\title{
Cognitive load and semantic analogies: Searching semantic space
}

\author{
Jean-Pierre Thibaut, Robert French, and Milena Vezneva \\ LEAD-CNRS and University of Burgundy, Dijon, France
}

\begin{abstract}
The aim of the present study is to investigate the performance of children of different ages on an analogymaking task involving semantic analogies in which there are competing semantic matches. We suggest that this can best be studied in terms of developmental changes in executive functioning. We hypothesize that the selection of common relational structure requires the inhibition of other salient features, in particular semantically related matches. Our results show that children's performance in classic A : B :: C : D analogy-making tasks seems to depend crucially on the nature of the distractors and the association strength between both the A and B terms and the $\mathrm{C}$ and $\mathrm{D}$ terms. These results agree with an analogy-making account (Richland, Morrison, \& Holyoak, 2006) based on different limitations in executive functioning at different ages.
\end{abstract}

Analogy-making is one of the most important ways in which adults and children make sense of their world. Extensive work suggests that analogy-making, in the sense of understanding and/or generating relations between objects or situations in the world, is a cognitive ability that develops gradually (Gentner, 1988; Goswami, 1992). In the present article, we hope to achieve a degree of reconciliation between the two main explanations of the development of analogy-making. The first holds that children's development can be explained in terms of a gradual increase in their structured knowledge of the world (Goswami, 1992, 2001; Goswami \& Brown, 1990; Vosniadou, 1995). A second, contrasting line of research emphasizes the role and development of cognitive factors that come under the heading of "executive functions." The latter approach claims that young children's ability to make analogies is tied to improvements in their executive functions that allow them to better handle cognitive load as they grow older. Halford (1993) ties the ability to do analogy-making to the ability to handle the cognitive load of dealing with a number of relations simultaneously (see also Halford, Wilson, \& Phillips, 1998). More recently, Richland, Morrison, and Holyoak (2006) and Thibaut, French, and Vezneva (2010) have stressed the importance of cognitive constraints in analogy-making. We will consider semantic analogies of the A : B :: C : D type (e.g., dog-doghouse and bird-nest). We conceptualize analogy-making as a search in an open semantic space. The number of relations holding between any A-B pair is high-indeed, potentially unlimited-because, depending on the context, relations can arise that would not usually come to mind (see Chalmers, French, \& Hofstadter, 1992; French, 1995, 2008; Hofstadter \& the Fluid Analogies Research Group, 1995; Mitchell, 1993;
Murphy \& Medin, 1985; Schyns, Goldstone, \& Thibaut, 1998; Thibaut \& Schyns, 1995).

As a result, analogy-making almost certainly entails the use of various components of executive functions. For example, searching "analogy space" requires working memory in order to test various solutions that come to mind and to keep them activated. It also requires attention because one has to systematically search for various semantic descriptors that might provide a solution to the analogy. This also implies cognitive flexibility when various structures do not lead to an analogy and exploration must take place along other dimensions. Finally, inhibition is also required when salient associations that are not relevant to the analogy at hand come to mind.

Before we come to the relation between knowledge and executive functions, we want to distinguish between two interpretations of the knowledge approach. The first interpretation is the one central to the traditional knowledge-based explanation. We might call it the descriptive or explicit knowledge view (which refers to what we can tell about one or several entities - e.g., that dogs like bones, or that an amplifier works with electricity). In the traditional knowledge-based explanation, the main claim was that a child should solve an analogy as long as he or she understood the relation between $\mathrm{A}$ and $\mathrm{B}$ on the one hand and between $\mathrm{C}$ and $\mathrm{D}$ on the other hand. We wish to stress another dimension of knowledge not explicitly considered in the literature on the development of analogy-making: the association strength between items (e.g., Tulving \& Madigan, 1970), which refers to the fact that one word immediately comes to mind when another word is given (e.g., cow and milk) because the two words are strongly associated in long-term memory (i.e., strongly entrenched), whereas other terms are as- 
sociated less strongly, so that hearing A does not immediately bring to mind B (e.g., child and bed). Note that "knowing" the relation between two terms is often independent of their semantic relatedness (e.g., everybody knows that children sleep in beds, even though the terms are not strongly associated in memory). We feel that the association strength between $\mathrm{A}$ and $\mathrm{B}$ and between $\mathrm{C}$ and $\mathrm{D}$ is a crucial factor in order to solve the analogy task. In standard A : B :: C : ? analogies, it is often easy to discover the relation between $\mathrm{C}$ and $\mathrm{D}$ when the pairs $\mathrm{A}-\mathrm{B}$ and $\mathrm{C}-\mathrm{D}$ are strongly associated. We hypothesize that this difference in entrenchment between strongly and weakly associated terms has significant processing consequences related to executive functions. Indeed, when these associations are weaker, one has to search the space more thoroughly to find an appropriate relation that makes the overall analogy work because, for weaker associations, each term in a pair will activate many strongly associated terms that, in general, will be irrelevant to the analogy problem to be solved. It will, therefore, be necessary to inhibit the semantic distractors that automatically come to mind. Solving these "weak" analogies also requires more cognitive flexibility, because the probability of immediately coming up with the right relation is lower than in the case of strong analogies. Thus, overall, more relations need to be explored-requiring greater cognitive monitoring - when the items are weakly associated than when they are strongly associated. This difference in association strength (i.e., a less entrenched relation) means that children, having more limited cognitive resources than adults do, should find these analogies more difficult to do. This reasoning also applies to the number of distractors, since three semantically related distractors require more information to inhibit than a single distractor does. In other words, "mapping" requires more than accessing the obviously semantic dimensions of the items, especially when the solution is based on weakly associated terms - that is, when the search space is broader. Richland et al. (2006) used scene analogy problems consisting of pairs of scenes illustrating relations among objects. The authors manipulated featural distraction by varying the identity of an object in the second scene of each pair. If the base scene included a running cat as part of the relation (i.e., dog chases cat), they added to the target scene a distractor object (i.e., an object not part of the chase relation) that was either perceptually similar (a sitting cat) or dissimilar to the object of the chase relation in the base scene (a sandbox). Results revealed that stimuli with similar distractors elicited more errors than the stimuli with dissimilar distractors.

Our view sharply contrasts with that of Leech, Mareschal, and Cooper (2008). According to these authors, solving an A : B :: C : ? analogy involves first extracting an a priori relation, $R$, between $\mathrm{A}$ and $\mathrm{B}$ and then applying this relation to $\mathrm{C}$. This so-called "relational priming" view depends on the existence of context-independent features and relations.

\section{Goals of the Present Article}

In what follows, we use the A : B :: C : D forced choice paradigm from Goswami and Brown (1990). We will focus on the interaction between associative strength and the number of distractors. In Goswami and Brown, children were shown drawings depicting A, B, C, and a set of possible solutions for D. Both the A and B and the $\mathrm{C}$ and $\mathrm{D}$ pairs were often closely related semantically (as shown by adults' ratings in our laboratory). Because of this, in order to select the analogical match to $\mathrm{C}$, the presence of the A : B pair was frequently unnecessary; for example, the A : B images were bird: nest and the $\mathrm{C}$ image was dog. There were four possible choices for D: doghouse (relational solution), bone, cat, and [different] dog. But the a priori semantic association between $d o g$ and $d o g$ house is strong enough to elicit the choice of doghouse a high percentage of the time, whether or not this was preceded by bird : nest. This was borne out by a test condition in which children were asked to find a picture among a set of four possible solutions that went with C (no A : B items were present). A significant number of semantically related choices were selected, but a comparable number of analogical matches were also chosen; see Figure 1 in Goswami and Brown.

We conjectured that using less semantically related analogical matches would force children to rely more on the relation between the initial $\mathrm{A}: \mathrm{B}$ pair, requiring them to move back and forth between this pair and the possible target pairs in order to find the most appropriate mappings (see Table 1 for a list of the problems used in our study). This exploration is particularly important if mapping is seen as a process in which the structures and attributes in

Table 1

Set of Analogy Problems and Distractors

\begin{tabular}{|c|c|c|c|c|c|c|}
\hline \multicolumn{4}{|c|}{ One Semantic Distractor } & \multicolumn{3}{|c|}{ Three Semantic Distractors } \\
\hline Analogy Problem & Solution & $\begin{array}{l}\text { Semantic } \\
\text { Distractor }\end{array}$ & $\begin{array}{l}\text { Unrelated } \\
\text { Foils }\end{array}$ & Analogy Problem & Solution & Semantic Distractors \\
\hline \multicolumn{7}{|c|}{ Weak Association } \\
\hline $\begin{array}{l}\text { wolf : meat :: goat : ? } \\
\text { man : plate :: pig :? } \\
\text { bird : plane :: fish :? }\end{array}$ & $\begin{array}{l}\text { grass } \\
\text { trough } \\
\text { boat }\end{array}$ & $\begin{array}{l}\text { horns } \\
\text { pig's tail } \\
\text { fisherman }\end{array}$ & $\begin{array}{l}\text { envelope, vase } \\
\text { key, pen } \\
\text { sofa, glasses }\end{array}$ & $\begin{array}{l}\text { child : bed :: cat : ? } \\
\text { jacket : wardrobe }:: \text { ring }: ? \\
\text { dress : hanger :: coat :? }\end{array}$ & $\begin{array}{l}\text { basket } \\
\text { box } \\
\text { hook }\end{array}$ & $\begin{array}{l}\text { mouse, whiskers, claws } \\
\text { finger, necklace, watch } \\
\text { scarf, sleeve, sweater }\end{array}$ \\
\hline \multicolumn{7}{|c|}{ Strong Association } \\
\hline $\begin{array}{l}\text { spider : web }:: \text { bee }: ? \\
\text { bird : nest :: dog : ? } \\
\text { train : rail :: boat :? }\end{array}$ & $\begin{array}{l}\text { hive } \\
\text { doghouse } \\
\text { water }\end{array}$ & $\begin{array}{l}\text { flower } \\
\text { bone } \\
\text { marine }\end{array}$ & $\begin{array}{l}\text { motorcycle, frame } \\
\text { guitar, apple } \\
\text { stool, hat }\end{array}$ & $\begin{array}{l}\text { cow }: \text { milk }:: \text { hen }: ? \\
\text { glove : hand :: shoe }: ? \\
\text { hen : chick :: horse }: ?\end{array}$ & $\begin{array}{l}\text { egg } \\
\text { foot } \\
\text { foal }\end{array}$ & $\begin{array}{l}\text { comb, grain, fox } \\
\text { sock, lace, sandal } \\
\text { saddle, stable, mane }\end{array}$ \\
\hline
\end{tabular}


the base and target domain gradually become available to processing (French, 1995; Hofstadter \& the Fluid Analogies Research Group, 1995; Mitchell, 1993).

Therefore, we manipulated two factors-the associative strengths between the $\mathrm{A}: \mathrm{B}$ and $\mathrm{C}: \mathrm{D}$ pairs, and the number of semantically related distractors, one or three. We expected that the use of relational choices with a weaker semantic relation to $\mathrm{C}$, combined with the presence of more semantic distractors, would make the analogies harder to solve and would lead to more errors, because processing loads would be increased. With weaker semantic relation, the solution does not come to mind immediately and requires that children explore each of the options more carefully. We ensured that children understood the relation between $\mathrm{A}$ and $\mathrm{B}$ and between $\mathrm{C}$ and $\mathrm{D}$. The presence of three semantic distractors would mean that a greater number of semantically related items would have to be inhibited than would be with a single distractor, thus leading to more errors in the former case. These predictions contrast with the standard "knowledge" view that posits that the number of semantic distractors should have only marginal effects once the child possesses the semantic knowledge relating $\mathrm{A}$ and $\mathrm{B}$ as well as $\mathrm{C}$ and D (Goswami \& Brown, 1990). Similarly, for the relational priming hypothesis (Leech et al., 2008) that equates analogy-making with a priming phenomenon, where the relational prime arises directly and unambiguously from A : B. Once the relation between A and B is understood, the number of semantic distractors should have little effect on children's responses. This might be the case for

Weak Association, One Semantic Distractor
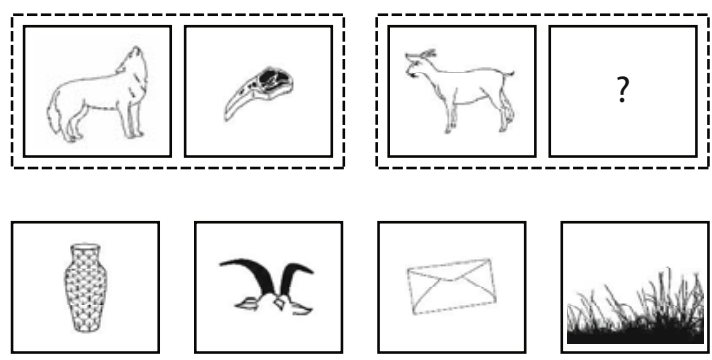

Semantic
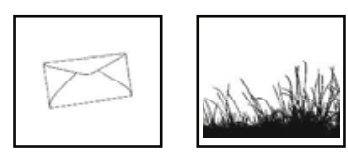

Unrelated

Analogical

Strong Association, One Semantic Distractor
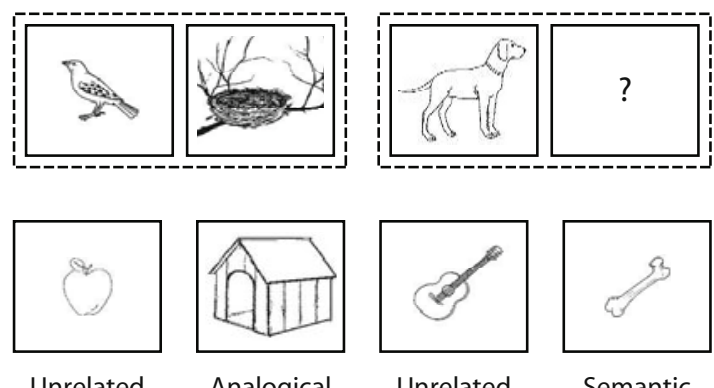

Unrelated

Analogical

Unrelated

Semantic

strongly semantically related A : B pairs, but it is not the case for less strongly associated pairs.

\section{The Present Experiment}

We used the traditional A : B :: C : ? analogy-making paradigm - that is, one in which the "relational" choice is the more valid one. We called the first pair (A : B) the base pair, and the pair $(\mathrm{C}:$ ? ) the target pair. The third stimulus (C) had to be matched with a fourth stimulus (D), such that the relation between $C$ and D was "the same as" the relation between A and B (see Figure 1). The material and instructions were patterned after Goswami and Brown (1990).

This experiment was a $2 \times 2 \times 2$ mixed design with age (4-year-olds and 5-year-olds) as a between-subjects factor, and association strength (strong vs. weak) and number of semantic distractors (one or three) as within-subjects factors. The dependent variable was the number of correct relational matches (see below for details).

\section{METHOD}

\section{Participants}

A total of 41 children took part in this experiment: twenty-two 4-year-old children ( $M=52$ months $)$ and nineteen 5-year-old children ( $M=67$ months) participated in the experiment. Informed consent was obtained from their parents.

\section{Materials}

The experiment consisted of a total of 13 trials divided into 1 practice trial and 12 experimental trials (see Table 1). Each of the

Weak Association, Three Semantic Distractors
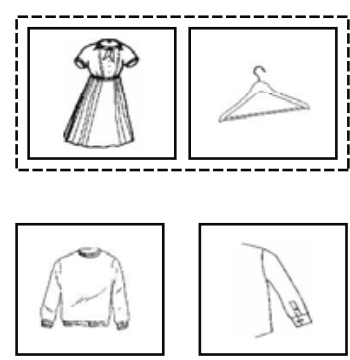

Semantic

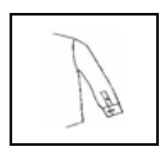

Semantic
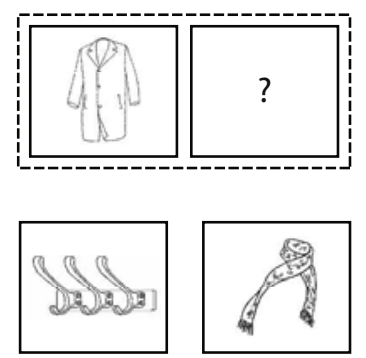

Analogical

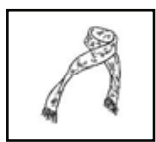

Semantic
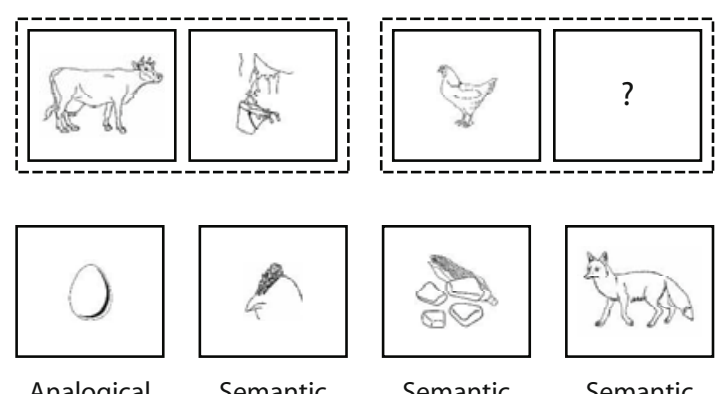

Semantic

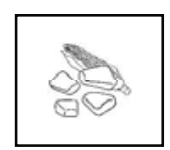

Semantic

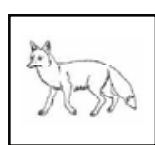

Semantic

Figure 1. Example of the four types of analogies used—-that is, weak versus strong, combined with one versus three distractors. 
four conditions consisted of 3 trials. Each trial contained seven drawings - namely, the items corresponding to the A, B, and C terms, and the four drawings shown as the solution set, consisting of the analogical match and three semantically related distractors (in the three-distractor condition) or the analogical match, one distractor, and two items that were semantically unrelated to the $\mathrm{C}$ term (in the one-distractor condition). (See Figure 1.) The strength of the semantic association between pairs of words with their corresponding picture used in the experiment was determined by 80 university students, who were asked to rate to what extent each item in the pair made them think of the other one. It was stressed that the task was to rate how strongly the two items were associated in their mind. The ratings were on a 1-7 scale. The strongly associated trials were composed of strongly associated A-B and C-D pairs, and the weakly associated trials were composed of weakly associated $\mathrm{A}-\mathrm{B}$ and $\mathrm{C}-\mathrm{D}$ pairs. We also rated the association strength between $\mathrm{C}$ and each of the semantically related distractors. The association strength of the weak A-B pairs fell between 2 and 3.5 (out of $7 ; M=2.69, S D=1.47$ ), whereas the association strength of the six strong pairs was between 6 and $6.50(M=6.114, S D=1.13)$. The $\mathrm{C}-\mathrm{D}$ association strength was between 3.35 and 4.75 for the weak items $(M=4.1, S D=1.44)$ and between 5.65 and 6.4 for the strong items $(M=6.05, S D=0.92)$.

The association strength between $\mathrm{C}$ and the distractors did not differ significantly from the association strength between $\mathrm{C}$ and $\mathrm{D}$. This strength was between 2.9 and 5.5 in the weak case $(M=4.38, S D=$ $1.31)$ and between 4.1 and 6.3 in the strong distractor case $(M=$ $5.65, S D=1.1) .{ }^{1}$ We controlled the stimuli to ensure that the strong condition would not be easier because the association strength between $\mathrm{C}$ and $\mathrm{D}$ was higher than the $\mathrm{C}$-distractor association strength in the strong condition and lower in the weak condition.

\section{Procedure}

The children were instructed to play a game in which they would be choosing pictures that went together. When they saw the picture cards, they were asked to give their name and to describe them. When they did not know the object's name, they were asked to describe it. The children knew $95 \%$ of the names, and when they did not know them, in most of the cases, they could give a description showing that they knew the stimulus. Overall, the percentage of cases in which children could neither name nor explain the item was less than $1 \%$. In these cases, the experimenter gave the children the missing information. The children saw the A : B pair and the $\mathrm{C}$ item in an array with the first two items grouped together to the left. The $\mathrm{C}$ item was alone on the right. Next to the $\mathrm{C}$ item there was a box containing a question mark for the solution item. The children studied these items without seeing the target items. They were then shown the four target items and were asked to point to the one that completed the series of items (cf. Goswami \& Brown, 1990). They were asked to justify their choice for each trial.

In the second part of the experiment, children's understanding of the semantic relation between $\mathrm{A}$ and $\mathrm{B}$ and between $\mathrm{C}$ and $\mathrm{D}$ was assessed. They were shown the A : B pairs and were asked to explain why the two pictures of each pair went well together. The same was true for the $\mathrm{C}$ : $\mathrm{D}$ pairs.

\section{RESULTS}

Performance was measured as the percentage of valid relational (i.e., most obvious relational) match. We eliminated all trials in which the children did not understand the semantic relation either between the A-B terms or between the $\mathrm{C}-\mathrm{D}$ terms, to be sure that children did not fail from a lack of the relevant knowledge for some of these pairs. Most of the difficulties in identifying the relations involved one trial in the weak association with one distractor-namely, the bird-plane : fish-boat associations. We, therefore, analyzed the data without this trial.
The percentage of trials that were eliminated for either failures to identify or misidentifications was $5 \%$ for the weak relations ( 6 for the 4 -year-olds and 4 for the 5 -yearolds; i.e., 2 per item) and $8 \%$ for the strong relations (10 for the 4 -year-olds and 9 for the 5 -year-olds; i.e., 3 per item). These were spread evenly across trials.

We ran a three-way mixed ANOVA on the data with age as a between-subjects factor and association strength (strong vs. weak) and number of semantic distractors (one or three) as within-subjects factors. The ANOVA revealed three significant main effects and an interaction between association strength and number of distractors. As expected, 5-year-olds performed better than 4-year-olds $\left[F(1,39)=8.79, p=.01, \eta_{\mathrm{p}}^{2}=.18\right]$, performance was significantly better on analogies with strong $\mathrm{A}-\mathrm{B}$ and $\mathrm{C}-\mathrm{D}$ relations than on those with weak $\mathrm{A}-\mathrm{B}$ and $\mathrm{C}-\mathrm{D}$ relations $\left[F(1,39)=17.55, p<.001, \eta_{\mathrm{p}}^{2}=.31\right]$, and performance was better on analogies with one semantic distractor than on those with three semantic distractors $[F(1,39)=8.20$, $\left.p<.01, \eta_{\mathrm{p}}^{2}=.17\right]$. Most importantly, however, the interaction between association strength and number of distractors was also significant $[F(1,39)=4.86, p<.03$, $\left.\eta_{\mathrm{p}}^{2}=.11\right]$ (Figure 2). A post hoc analysis (Tukey HSD) revealed that the only significant difference was between the condition "weak-three distractors" and the three other conditions $(p<.01)$

We also analyzed the type of distractors chosen in the condition with one semantic distractor. In the weak- and strong-association conditions, there were $81 \%$ and $82 \%$ of semantic errors, respectively, where chance level was at $33 \%$.

\section{DISCUSSION}

We manipulated the number of distractors and the one dimension of semantic knowledge that has been neglected so far in the literature on analogy-making. The main factors had a significant effect. There is a significant main effect of age between the 4- and 5-year-old children. Also, analogies with strongly associated items in the base and target pairs produced better performance than those with weakly associated items in the base and target pairs; fi-

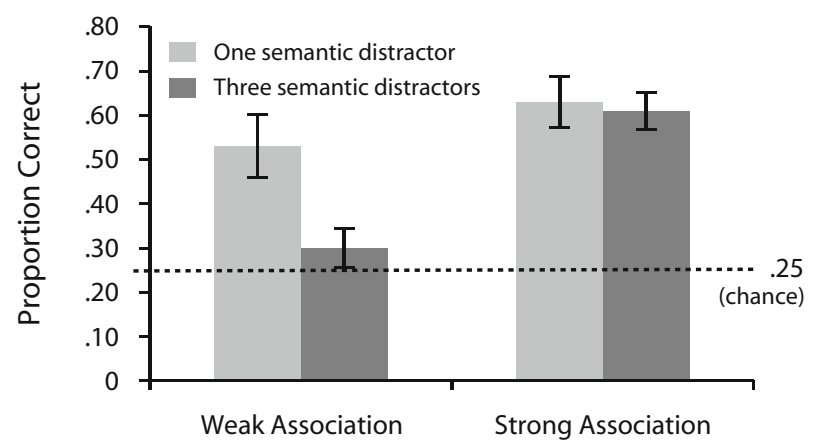

Figure 2. Interaction between association and number of semantic distractors. The only significant difference was between the weak associations in the three-distractor case and the other conditions. Note that there was no difference between the weak one-distractor and the strong trials. 
nally, the effect of the number of distractors on performance was significant.

The key finding, however, is the significant interaction between association strength and number of distractors. This shows that increasing the number of semantic distractors has a more deleterious effect when the items of the A : B pair and C : D pair are weakly associated (e.g., man : plate :: pig : trough) than when they are strongly associated (e.g., spider : web :: bee : hive).

This result makes perfect sense in the framework of a cognitive load and search space hypothesis, which predicts greater difficulties from numerous semantic competitors when the associations are weak. When the choices in the semantic search space are not dominated by a strongly associated base pair and target pair, the search for an appropriate answer becomes more difficult for two reasons: (1) because more search is required, and (2) because, when several semantic competitors are available during the search, they must be inhibited in order to come up with the (unique) solution.

This result contrasts with what we called the "descriptive interpretation" of the knowledge hypothesis (e.g., Goswami \& Brown, 1990; Rattermann \& Gentner, 1998). According to this theory, there would be no clear reason that increasing the number of semantic distractors or decreasing the a priori semantic association strength between the analogy components would dramatically lower children's performance. The same is true for Leech et al.'s (2008) relational priming hypothesis. As soon as the relation $R$ between $\mathrm{A}$ and $\mathrm{B}$ has been noticed, it would be applied to $\mathrm{C}$ and the solution found. The number of distractors should not matter; yet we clearly see that children's performance, in fact, did decrease significantly when the association strengths between items were weaker and when there were more semantic distractors. Neither the relational priming view nor the "descriptive" knowledge-based view would seem to be able to adequately account for the difference in error rates between the one- and three-distractor conditions in the weak case.

As for the role of the semantic strength of the associations involved in an analogy, we believe that it is a major factor in solving an analogy problem. This is related to the associative strength version of the knowledge hypothesis (see the beginning of this article). If two concepts are strongly associated (i.e., the relation is deeply entrenched), one concept primes the other, and there is less need for executive control to find a solution. It is only when the relation is not deeply entrenched (i.e., the objects are weakly associated) that executive control is necessary to explore the space of possible solutions for an answer. This is when distractors have a significant influence on performance, because, during this exploration, they are considered to be potential solutions to the problem, thus leading to more errors.

These results support the notion of the fundamental role in children's analogy-making of their developing ability to handle cognitive load. Like adults, when confronted with an analogy they look for potential solutions or construct these solutions by testing various hypoth- eses (i.e., features and relations between features). When there is no obvious solution, they construct and compare different possible solutions and gradually construct appropriate representations of the objects and the relations between them necessary to do the analogy. To find a good solution, they have to inhibit other salient but less appropriate solutions and be flexible enough to replace tentative solutions with ones that appear to be better, while remaining prepared to return to their initial choice later on. The point is that this process generates cognitive load associated with executive functioning (Richland et al., 2006; Thibaut et al., 2010).

In conclusion, we have presented work that demonstrates the role of association strength among items making up the base and target pairs of analogies. We have shown that children's performance is affected by the increased cognitive load of the number of semantically related distractors and the association strengths of the pairs involved in the analogy. A theory of analogy-making based on mechanisms of cognitive load appears to provide a relatively straightforward explanation of these data, whereas it is hard to see how other theories not based on cognitive load might explain these results.

\section{AUTHOR NOTE}

This research has been supported by European Commission Grant FP6-NEST-029088 and was presented at the 2009 SRCD Conference and the 2009 Cognitive Science Society Conference. Correspondence concerning this article should be addressed to J.-P. Thibaut, University of Burgundy, LEAD-CNRS UMR 5022, Pôle AAFE-Esplanade Erasme BP 26513,21065 Dijon Cedex, France (e-mail: jean-pierre.thibaut@ u-bourgogne.fr).

\section{REFERENCES}

Chalmers, D. J., French, R. M., \& Hofstadter, D. R. (1992). Highlevel perception, representation, and analogy: A critique of artificial intelligence methodology. Journal of Experimental \& Theoretical Artificial Intelligence, 4, 185-211.

French, R. M. (1995). The subtlety of sameness: A theory and computer model of analogy-making. Cambridge, MA: MIT Press.

FRENCH, R. M. (2008). Relational priming is to analogy-making as oneball juggling is to seven-ball juggling. Behavioral \& Brain Sciences, 31, 386-387.

Gentner, D. (1988). Metaphor as structure mapping: The relational shift. Child Development, 59, 47-59.

Goswami, U. (1992). Analogical reasoning in children. Hillsdale, NJ: Erlbaum.

Goswami, U. (2001). Analogical reasoning in children. In D. Gentner, K. J. Holyoak, \& B. N. Kokinov (Eds.), The analogical mind: Perspectives from cognitive science ( $\mathrm{pp}$. 437-470). Cambridge, MA: MIT Press, Bradford Books.

Goswami, U., \& Brown, A. L. (1990). Higher-order structure and relational reasoning: Contrasting analogical and thematic relations. $\mathrm{Cog}$ nition, 36, 207-226.

Halford, G. S. (1993). Children's understanding: The development of mental models. Hillsdale, NJ: Erlbaum.

Halford, G. S., Wilson, W. H., \& Phillips, S. (1998). Processing capacity defined by relational complexity: Implications for comparative, developmental, and cognitive psychology. Behavioral \& Brain Sciences, 21, 803-831.

Hofstadter, D. R., \& the Fluid Analogies Research Group (1995). Fluid concepts and creative analogies: Computer models of the fundamental mechanisms of thought. New York: Basic Books.

Leech, R., Mareschal, D., \& Cooper, R. (2008). Analogy as relational priming: A developmental and computational perspective on 
the origins of a complex cognitive skill. Behavioral \& Brain Sciences, 31, 375-378.

Mitchell, M. (1993). Analogy-making as perception: A computer model. Cambridge, MA: MIT Press.

Murphy, G. L., \& Medin, D. L. (1985). The role of theories in conceptual coherence. Psychological Review, 92, 289-316.

Rattermann, M. J., \& Gentner, D. (1998). More evidence for a relational shift in the development of analogy: Children's performance on a causal-mapping task. Cognitive Development, 13, 453-478.

Richland, L. E., Morrison, R. G., \& HolyoaK, K. J. (2006). Children's development of analogical reasoning: Insights from scene analogy problems. Journal of Experimental Child Psychology, 94, 249-273.

Schyns, P. G., Goldstone, R. L., \& Thibaut, J.-P. (1998). The development of features in object concepts. Behavioral \& Brain Sciences, 21, 1-54.

Thibaut, J.-P., French, R. M., \& Vezneva, M. (2010). Analogymaking in children: The importance of processing constraints. Journal of Experimental Child Psychology, 1, 1-19.

Thibaut, J.-P., \& Schyns, P. G. (1995). The development of feature spaces for similarity and categorization. Psychologica Belgica, 35, 167-185.

Tulving, E., \& Madigan, S. E. (1970). Memory and verbal learning. Annual Review of Psychology, 2, 437-484.

VosNiadou, S. (1995). Analogical reasoning in cognitive development. Metaphor \& Symbol, 10, 297-308.

\section{NOTE}

1. There were two exceptions to this. In the three-distractor trial weak case, with cat-basket (C-D), cat-mouse (5.2) was higher than the association between $\mathrm{C}$ and D. However, the association between the two other distractors did not differ significantly from the association between $\mathrm{C}$ and $\mathrm{D}$. In the strong case, the $\mathrm{C}-\mathrm{D}$ association hen-egg (a threedistractor trial) was significantly higher than between $\mathrm{C}($ hen $)$ and the distractor fox.

(Manuscript received August 4, 2009;

revision accepted for publication February 17, 2010.) 